For the purpose of estimating the angular sizes of the burst sources, six Jovian noise storms were examined. Two of these were recorded with a receiver bandwidth of $4 \mathrm{kc} / \mathrm{s}$ and four with a bandwidth of $200 \mathrm{kc} / \mathrm{s}$. From the narrowband records, analysis of 35 bursts led to a value of $\bar{V}_{n}=1.00$ for the mean fringe visibility, with an S.E. of 0.03 . From the wide-band interferometer, 35 bursts resulted in a mean fringe visibility of $V_{w}=$ 1.04, with an S.E. of 0.06 . Much of the larger error in the wide-band result may be due to the necessity of applying corrections to the observed fringe visibilities for band-width dispersion. Consideration of the standard errors involved in these mean values leads to estimates of an upper size limit for the burst-emitting sources. From

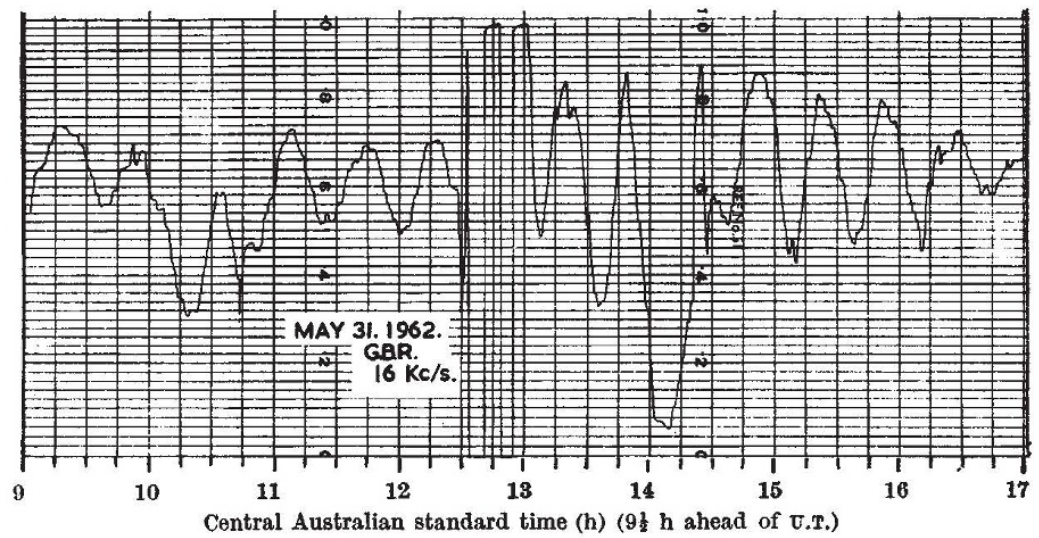

Central Australian standard time (h) $\left(9 \frac{1}{2} \mathrm{~h}\right.$ ahead of U.T.)

Fig. 1 the narrow-band results, for a Gaussian brightness distribution, the angular size of the sources was $\leqslant 13^{\prime \prime}$ are at the 5 per cent confidence-level. The wide-band result indicates that the source size was $\leqslant 19^{\prime \prime}$ arc. There were no detectable systematic differences in source size between the polar and equatorial directions.

Size of the burst-emitting regions. Restricting consideration to those observations for which the absence of marked ionospheric effects results in a high degree of correlation between the total power records at both sites, it is possible to set an upper limit on the size of the region responsible for the bursts in a storm. In these circum. stances and where the bursts are sufficiently frequent, they give rise to a continuous series of fringes. For example, during a storm on September 25, 1962, 27 such fringes were counted over a period of $9 \mathrm{~min}$. The mean fringe period was 19.8 sec with an $S . E$. of 12 per cent for individual measurements. Hence it was most unlikely (at the 5 per cent confidence-level) that independently radiating sources, if indeed there was more than a single source, occupied an area with dimensions greater than $\frac{1}{4}$ of the fringe separation or $25^{\prime \prime}$ arc. A number of such occurrences showed that there were no differences between equatorial and polar directions.

There seems to be no reason, from the results recorded here, why interferometer observations should not be continued with longer baselines until the Jovian burst sources are resolved. It is our present intention to make similar measurements over baselines of $80 \mathrm{~km}$ or more luring the 1963 opposition.

We thank Mr. C. F. Fryar for assistance in operating the interferometer during the present experiment.

O. B. Sleet

C. S. Higarns

Commonwealth Scientific and

Industrial Research Organization,

Radiophysics Laboratory, Sydney, Australia.

\section{Sudden Changes in Amplitude and Phase of the Very-Low-Frequency Signal from \\ Station GBR at Salisbury, South Australia}

A PECuLrar phenomenon, to our knowledge not reported so far, has been observed on the signals of Station $G B R$ on $16 \mathrm{kc} / \mathrm{s}$.

The signals of this Station, monitored for frequency standardization purposes, are mixed with a suitable frequency derived from a $100-\mathrm{kc} / \mathrm{s}$ standard oscillator and a record, as the one shown in Fig. 1, is produced. The number of beat cycles over 24 -h intervals is counted and the drift of the local source determined.

At about $12.30 \mathrm{~h}$ local time ( $9 \frac{1}{2} \mathrm{~h}$ ahead of U.T.) on May 31,1962 , the number of beat cycles suddenly increased. and this faster beat persisted for about half an hour, as shown in the figure.
Similar occurrences were recorded on June 11 at approximately $12.30 \mathrm{~h}$ local time, on June 12 at $09.45 \mathrm{~h}$ and on June 25 at $09 \cdot 40 \mathrm{~h}$. These lasted approximately 15, 30 and $8 \mathrm{~min}$ respectively.

All possibility of instability of the local source must be ruled out as the same derived signal is mixed also with the signals of the United States transmitter $N B A$ on $18 \mathrm{kc} / \mathrm{s}$ and no trace of a similar effect has been noticed on the record of this station.

The beat frequency, while the described phenomenon lasted, was of a high stability not likely to be possible to derive from a local source switched on for this period.

We thank the Chief Scientist, Department of Supply, for his permission to publish this communication.
P. ROHAN
L. L. ANDERSON
D. J. Cooke

Electronic Techniques Group,

Weapons Research Establishment, Salisbury, South Australia.

\section{GEOPHYSICS}

\section{Lithium Emission in Twilight at Uppsala during November 1962}

A PATRoL spectrophotometer (SP3) has been operating at this Observatory for $a$ long period to record lithium emission in twilight at $6708 \AA$. In spite of the high sensitivity of the instrument no lithium was recorded until a strong emission suddenly occurred on November 7, 1962, in the evening twilight. The last record before this date was made on October 19, 1962, when no emission at all was detected. In the days before and after November 7 the weather conditions were unfavourable, but in spite of this a number of good records have been received.

The spectrophotometer was adjusted on November 7 to sweep over a range of $\sim 50 \AA$ each fourth minute, and the spectra] line is recorded via photomultiplier and rate. meter on an $x-y$ recorder. The resolution was about $2.5 \AA$ and the instrument was directed $25^{\circ}$ elevation north. north-west. Fig. I shows the first record of the lithium flash.

After November 7, records were made in zenith both in the morning and evening twilight. Simultaneous records were made with another spectrophotometer of somewhat less light power including the sodium lines for comparison. From these records one can get some idea of the ratio and the time difference of the lithium flash with respect to the sodium flash. Regarding the intensity of the lithium emission a comparison with sodium shows that the lithium emission at the beginning of the period seems to have been at least of the same order as the sodium emission. Regard. ing the time difference with respect to sodium, the lithium flash follows very near that of sodium, but both are displaced in time with respect to the $O I 6300 \AA$ flash, which 